\title{
Aortic stiffness and ambulatory blood pressure as predictors of diabetic kidney disease: a competing risks analysis from the Rio de Janeiro Type 2 Diabetes Cohort Study
}

\author{
Claudia R. L. Cardoso ${ }^{1} \cdot$ Nathalie C. Leite $^{1} \cdot$ Guilherme C. Salles $^{2} \cdot$ Marcel T. Ferreira $^{1}$ • \\ Gil F. Salles ${ }^{1}$
}

Received: 27 May 2017 / Accepted: 28 September 2017 /Published online: 23 October 2017

(C) Springer-Verlag GmbH Germany 2017

\begin{abstract}
Aims/hypothesis Diabetic kidney disease (DKD) is a microvascular complication associated with poor control of blood glucose and BP. We aimed to evaluate the predictors of development and progression of DKD in a cohort of high-risk individuals with type 2 diabetes, placing emphasis on ambulatory BP and arterial stiffness.

Methods In a prospective study, 629 individuals without advanced renal failure had their renal function evaluated annually over a median follow-up period of 7.8 years. Ambulatory BP was monitored and aortic stiffness was assessed by carotid-femoral pulse wave velocity at baseline. Multivariate competing risks analysis with all-cause mortality, using the Fine and Gray approach, was used to examine the independent predictors of development and progression of $\mathrm{DKD}$, a composite of development or progression of abnormal albuminuria and worsening of renal function (doubling of serum creatinine or progression to end-stage renal disease).

Results At baseline, 197 individuals had DKD. During followup, DKD developed or progressed in 195 individuals, abnormal albuminuria developed or progressed in 125 individuals and renal function deteriorated in 91 . After adjustments for baseline
\end{abstract}

Electronic supplementary material The online version of this article (https://doi.org/10.1007/s00125-017-4484-z) contains peer-reviewed but unedited supplementary material, which is available to authorised users.

Gil F. Salles

gilsalles@ hucff.ufrj.br

1 Department of Internal Medicine, University Hospital Clementino Fraga Filho, School of Medicine, Universidade Federal do Rio de Janeiro, Rua Rodolpho Paulo Rocco, 255, Cidade Universitária, Rio de Janeiro CEP: 21941-913, Brazil

2 Civil Engineering Program, COPPE, Universidade Federal do Rio de Janeiro, Rio de Janeiro, Brazil albuminuria and renal function, age, sex, diabetes duration and use of renin-angiotensin antagonists, poorer control of blood glucose (HR 1.17; 95\% CI 0.98, 1.40; $p=0.09$ for each $1 \mathrm{SD}$ increment in mean first-year $\mathrm{HbA}_{1 \mathrm{c}}$ ), higher ambulatory systolic BP (HR 1.28; 95\% CI 1.09, 1.50; $p=0.003$, for each 1 SD increase in daytime systolic BP $[\mathrm{SBP}])$ and increased aortic stiffness (HR 1.16; 95\% CI 1.00, 1.34; $p=0.05$ ) were independent predictors of development or progression of DKD. At baseline, ambulatory BP was a stronger predictor than BP measured in the clinic. Aortic stiffness predicted abnormal albuminuria development or progression (HR 1.26; 95\% CI 1.02, 1.56; $p=0.036$ ) whereas ambulatory BP was a stronger predictor of renal function deterioration (HR 1.32; 95\% CI 1.09, 1.60; $p=0.005$ for daytime SBP).

Conclusions/interpretation Poor blood glucose and BP control and increased aortic stiffness were the main predictors of development or progression of DKD; ambulatory SBP was a better predictor than BP measured in the clinic. Ambulatory BP monitoring and assessment of aortic stiffness should be more widely used in clinical type 2 diabetes management.

Keywords Albuminuria - Ambulatory blood pressures · Aortic stiffness · Diabetic kidney disease $\cdot$ Type 2 diabetes

$\begin{array}{ll}\text { Abbreviations } \\ \text { ACEi } & \text { Angiotensin-converting enzyme inhibitor } \\ \text { ARB } & \text { Angiotensin II receptor blocker } \\ \text { cf-PWV } & \text { Carotid-femoral pulse wave velocity } \\ \text { CKD-EPI } & \text { Chronic Kidney Disease Epidemiology } \\ & \text { Collaboration } \\ \text { DBP } & \text { Diastolic BP } \\ \text { DKD } & \text { Diabetic kidney disease } \\ \text { ESRD } & \text { End-stage renal disease } \\ \text { SBP } & \text { Systolic BP }\end{array}$




\section{Introduction}

Type 2 diabetes incidence is increasing worldwide [1] and currently diabetic kidney disease (DKD) is the main cause of end-stage renal disease (ESRD) [2,3]. In addition to increased morbidity and precocious mortality, there is a considerable social and economic burden and reduced quality of life associated with the presence and severity of chronic kidney disease in individuals with diabetes $[2,4]$.

Control of blood glucose levels and BP is of utmost importance for the prevention of diabetic microvascular complications. In particular, BP and blood glucose control is important for preventing development and progression of abnormal albuminuria in DKD [5-8]. However, whether intensive control hinders progression to ESRD in individuals with type 2 diabetes remains debatable [5-10]. The interaction between blood glucose control and BP control when assessing renal outcomes needs additional investigation; some studies show no additive benefit from combined treatment $[11,12]$ and others show an interaction at higher BPs $[8,13]$. Moreover, ambulatory BP has been demonstrated to be a superior cardiovascular risk marker when compared with $\mathrm{BP}$ measured in the clinic [14], including in individuals with type 2 diabetes [15]. However, few previous studies [16, 17] have investigated the prognostic impact of ambulatory BP in renal outcomes or compared ambulatory with clinic BP measurements in type 2 diabetes.

Central (aortic) arterial stiffness has been proposed as a surrogate for cumulative vascular risk factor burden [18]. Individuals with type 2 diabetes have increased aortic stiffness [19] and cross-sectional relationships between the presence of DKD and increased arterial stiffness have been demonstrated [20-22]. However, only two previous longitudinal studies, performed in Asian individuals, showed that aortic stiffness is associated with incident microalbuminuria and decrease in glomerular filtration rate $[23,24]$. To date, no occidental study has investigated these relationships.

Therefore, we intended to investigate the predictive factors for several renal outcomes in high-risk individuals with type 2 diabetes. We focused on evaluating the prognostic importance of aortic stiffness and comparing the value of clinic and ambulatory BPs as predictors for DKD development and progression, using primarily a competing risks analysis approach.

\section{Methods}

Participants The Rio de Janeiro Type 2 Diabetes Cohort Study was a prospective study in which 650 individuals with type 2 diabetes were recruited between August 2004 and December 2008 and re-evaluated annually for DKD until December 2015 in the diabetes outpatient clinic of our tertiary-care University Hospital. All participants gave written informed consent and the local Ethics Committee had previously approved the study protocol. The characteristics of this cohort, the baseline procedures and the diagnostic definitions have been described previously $[15,21,25,26]$. Briefly, inclusion criteria were as follows: adults up to 80 years old who had type 2 diabetes and either microvascular (retinopathy, nephropathy or neuropathy) or macrovascular (coronary, cerebrovascular or peripheral artery disease) complications, or who had at least two other modifiable cardiovascular risk factors. Exclusion criteria were morbid obesity (BMI $\geq 40 \mathrm{~kg} / \mathrm{m}^{2}$ ), stage IV or $\mathrm{V}$ chronic kidney disease (serum creatinine

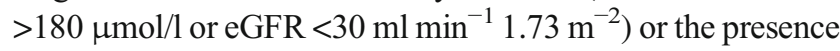
of any serious concomitant disease limiting life expectancy.

Baseline procedures All participants were submitted to a standard baseline protocol that included a thorough clinical examination, a laboratory evaluation, $24 \mathrm{~h}$ ambulatory BP monitoring and aortic stiffness assessment by carotid-femoral pulse wave velocity (cf-PWV). Diagnostic criteria for diabetic chronic complications were detailed previously $[15,21,25$, 26]. In brief, coronary heart disease was diagnosed by clinical, ECG criteria or by positive ischaemic stress tests. Cerebrovascular disease was diagnosed by history and physical examination and peripheral arterial disease by an anklebrachial index $<0.9$. Diabetic retinopathy was evaluated by a single specialist in retinal ophthalmology. The diagnosis of DKD needed at least two albumin excretion rate values $\geq 30 \mathrm{mg} / 24 \mathrm{~h}$, confirmed reduction of eGFR $\leq 60 \mathrm{ml} \mathrm{min}{ }^{-1}$ $1.73 \mathrm{~m}^{-2}$ (estimated by the Chronic Kidney Disease Epidemiology Collaboration [CKD-EPI] equation [27]) or serum creatinine $>130 \mu \mathrm{mol} / \mathrm{l}$, with the concomitant presence of diabetic retinopathy. Peripheral neuropathy was determined by standard clinical examination as previously detailed [25]. $\mathrm{BP}$ was measured in the clinic three times using a digital oscillometric BP monitor (HEM-907XL; Omron Healthcare, Kyoto, Japan) with a suitably sized cuff on two occasions 2 weeks apart at study entry. The first measure of each visit was discarded and the recorded BP was the mean of the last two readings at each visit. A diagnosis of arterial hypertension was made when mean systolic BP (SBP) $\geq 140 \mathrm{mmHg}$ or diastolic BP (DBP) $\geq 90 \mathrm{mmHg}$ or if antihypertensive drugs had been prescribed. Ambulatory BP was recorded in the following month using Mobil-O-Graph, version 12 equipment (Dyna-Mapa; Cardios, São Paulo, Brazil). Variables evaluated were $24 \mathrm{~h} \mathrm{BP}$, daytime and night-time BP and the nocturnal fall in BP (calculated as the percentage decrease in night-time $\mathrm{BP}$ in relation to daytime levels: [(daytime $\mathrm{BP}$ - night-time $\mathrm{BP}$ )/daytime $\mathrm{BP}] \times 100 \%$ ). A normal dipping pattern was defined as nocturnal fall in $\mathrm{BP} \geq 10 \%[15,28]$. Twenty-two untreated individuals with normal clinic BP but high ambulatory $\mathrm{BP}$ ( $24 \mathrm{~h} \mathrm{BP} \geq 130 / 80 \mathrm{mmHg}$ ) were also considered as hypertensive. 
Laboratory evaluations included fasting blood glucose, $\mathrm{HbA}_{1 \mathrm{c}}$, serum creatinine and lipids. Serum creatinine was measured at the University Hospital central laboratory by a modified kinetic Jaffe's method; values were multiplied by 0.95 because they were not standardised to isotope dilution mass spectrometry [29]. Albuminuria was evaluated by nephelometry in two non-consecutive sterile $24 \mathrm{~h}$ urine collections (lower detection limit $0.002 \mathrm{~g} / \mathrm{l}$; intra-assay and inter-assay variation coefficients 4.3 and $4.4 \%$, respectively) and was assessed annually during follow-up. Aortic stiffness was evaluated by measuring cf.-PWV, using the foot-to-foot velocity method with Complior equipment and software (ArtechMedical, Paris, France), as previously described [19, 23]. Direct carotid-femoral distance was corrected by a factor of 0.8 ; cf-PWV $>10 \mathrm{~m} / \mathrm{s}$ was considered to indicated increased aortic stiffness, as recommended [30].

Follow-up The participants were followed-up regularly at least three or four times a year and all had at least two to four measurements of annual $\mathrm{HbA}_{1 \mathrm{c}}$, serum lipids, serum creatinine and clinic BP. Mean values during each specific time interval were recorded, as well as mean cumulative values during the whole follow-up period, until endpoint occurrence or censoring (administrative or because of death). No participants were lost to follow-up, except the deceased individuals.

\section{Outcomes and assessment of development and progres-} sion of DKD The primary endpoint was a composite of abnormal albuminuria development/progression and worsening in outcomes of renal function. The endpoint for development and progression of albuminuria was defined, respectively, as a change from normal (albumin excretion rate $<30 \mathrm{mg} / 24 \mathrm{~h}$ ) to microalbuminuria (30-299 $\mathrm{mg} / 24 \mathrm{~h}$ ) and a change from microalbuminuria to macroalbuminuria ( $\geq 300 \mathrm{mg} / 24 \mathrm{~h}$ ) on at least two consecutive $24 \mathrm{~h}$ measurements of urinary albumin excretion over a period of 3-6 months at any annual examination. The endpoint for worsening renal function was a doubling of serum creatinine to a level of $\geq 200 \mu \mathrm{mol} / \mathrm{l}(\geq 2.3 \mathrm{mg} / \mathrm{dl})$ on at least two consecutive visits (3-4 months' interval) together with no improvement after a minimum interval between examinations of 6 months, or an occurrence of ESRD requiring dialysis, renal transplantation or death due to renal failure.

Statistical analysis Continuous data are described as means \pm SD or as medians (interquartile range). Data from participants with and without occurrence of any renal endpoint during follow-up were compared by unpaired $t$ test (for continuous normal variables), Mann-Whitney test (for continuous asymmetric variables) and by $\chi^{2}$ test (for categorical variables). The independent predictors of an endpoint's occurrence were primarily examined by competing risks (with all-cause mortality) analyses, using the proportional subdistribution hazards model (Fine and Gray approach) [31]. The cumulative incidence function was used to estimate the cumulative incidence of endpoints during follow-up for the renal outcomes and for the competing event. Additionally, classic Cox proportional hazards analysis was performed as a secondary analysis. The renal outcomes were assessed as a composite endpoint, restricted to the first incident event, and also as separate endpoints (abnormal albuminuria development/progression and worsening of renal function). The following candidate variables were used for entry into the multivariate analyses, based on biological plausibility: BMI, smoking status, physical activity, glucose-lowering treatment, antihypertensive treatment (number and classes of drugs), presence of any macrovascular (coronary, cerebrovascular and peripheral arterial disease) and microvascular (retinopathy, neuropathy) diabetic complications at baseline, aortic stiffness, clinic and ambulatory BPs, $\mathrm{HbA}_{1 \mathrm{c}}$, HDL-cholesterol and LDL-cholesterol. Clinic BPs and $\mathrm{HbA}_{1 \mathrm{c}}$ levels were examined as baseline values and as mean values during specific periods of follow-up. Age, sex, diabetes duration, baseline albumin excretion rate and eGFR and use of an angiotensin-converting enzyme inhibitor (ACEi) or angiotensin II receptor blocker (ARB) during follow-up were considered as background adjusting covariates for all analyses. First, each candidate variable was entered separately into the background adjusted model, then those with an adjusted $p$ value $<0.10$ were used in the final predictive models for each outcome. Competing risks results are presented as subdistribution HRs (95\% CIs); to allow comparisons between the independent predictors, HRs were calculated for standardised increments of $1 \mathrm{SD}$ of the continuous variables. To assess the relative prognostic importance of each $\mathrm{BP}$ variable, clinic and ambulatory BPs were evaluated in separate competing risks models, adjusted for the same covariates of the original predictive model. To assess for possible effect modifications between blood glucose and BP control on occurrence of renal outcomes, interaction terms between $\mathrm{HbA}_{1 \mathrm{c}}$ and BPs were tested separately in all analyses, as well as interactions between the independent predictors and age, sex and baseline albuminuria and eGFR. To exclude the possibility of reverse causality between BP and progression of DKD, two additional sensitivity analyses were performed. First, we excluded from analysis those participants with preexistent DKD at baseline (197 individuals, 31\%). Hence, this analysis only evaluated new incidence of microalbuminuria and renal function worsening (as all participants had normal albuminuria and eGFR $>60 \mathrm{ml} \mathrm{min}^{-1} 1.73 \mathrm{~m}^{-2}$ at study entry). Second, we excluded from analysis those individuals who achieved any of the endpoints during the first 3 years of follow-up. This ensures that there was a minimum time interval of 2 years between baseline and first-year variables and assessment of renal outcomes. All the additional sensitivity analyses were performed within the competing risks framework. Statistics were performed with SPSS version 19.0 (SPSS, Chicago, IL, USA) and with R version 3.4.1, cmprsk 
package (R Foundation for Statistical Computing, Vienna, Austria); two-tailed $p$ value $<0.05$ was considered significant.

\section{Results}

Baseline characteristics and incidence of renal outcomes during follow-up Six-hundred and fifty individuals were evaluated at baseline, 13 died during the first year of follow-up and eight did not collect at least a second $24 \mathrm{~h}$ urine sample during follow-up (the median number of $24 \mathrm{~h}$ urine examinations was five per person [maximum of 12 per person]), leaving a total of 629 individuals for renal endpoint evaluation. At baseline, 152 participants (24.2\%) had microalbuminuria, 14 (2.2\%) had macroalbuminuria and 31 (4.9\%) had decreased renal function (eGFR $\leq 60 \mathrm{ml} \mathrm{min}^{-1} 1.73 \mathrm{~m}^{-2}$ ) without abnormal albumin excretion rate but with concomitant diabetic retinopathy.

After a median follow-up of 7.8 years (range $1-10.7$ years), 434 individuals (69.0\%) remained stable and 195 (31.0\%) developed one of the renal endpoints. Separately, 125 individuals $(19.9 \%)$ either developed microalbuminuria or progressed to macroalbuminuria. Of the 463 participants without abnormal albumin excretion rate at baseline, 100 (21.6\%) developed persistent microalbuminuria; none of the participants progressed to macroalbuminuria. Of the 152 participants who displayed microalbuminuria at baseline, 25 (16.5\%) progressed to macroalbuminuria, 24 (15.8\%) regressed to normoalbuminuria and the rest remained microalbuminuric. Otherwise, 91 of the 629 participants (14.5\%) developed a renal failure endpoint: the serum creatinine of 59 individuals doubled to a level of at least $200 \mu \mathrm{mol} / \mathrm{l}(2.3 \mathrm{mg} / \mathrm{dl})$; dialysis was initiated in 25 individuals and seven died from renal failure. Sixty-three participants died before achieving the microalbuminuria outcomes and 83 died from non-renal causes before reaching one of the renal failure outcomes, prompting the competing risks analyses. Figure 1 shows the cumulative incidence function and respective competing risk event (deaths from non-renal causes) for each endpoint.

Table 1 outlines the characteristics of the participants, grouped according to whether they presented a composite renal outcome. Participants presenting a composite renal outcome had longer diabetes duration and greater prevalence of other microvascular complications than those who did not. Those who developed DKD or whose DKD progressed used insulin more frequently and took a greater number of antihypertensive medications than the participants with no renal outcome. They also had greater clinic and ambulatory SBPs than those in whom DKD did not develop or progress. $\mathrm{HbA}_{1 \mathrm{c}}$ levels followed a similar pattern: except for baseline values, all $\mathrm{HbA}_{1 \mathrm{c}}$ levels during follow-up were higher in participants presenting a renal outcome. Participants with a renal endpoint had greater left ventricular mass index and higher aortic stiffness than those in whom DKD did not develop or progress. a

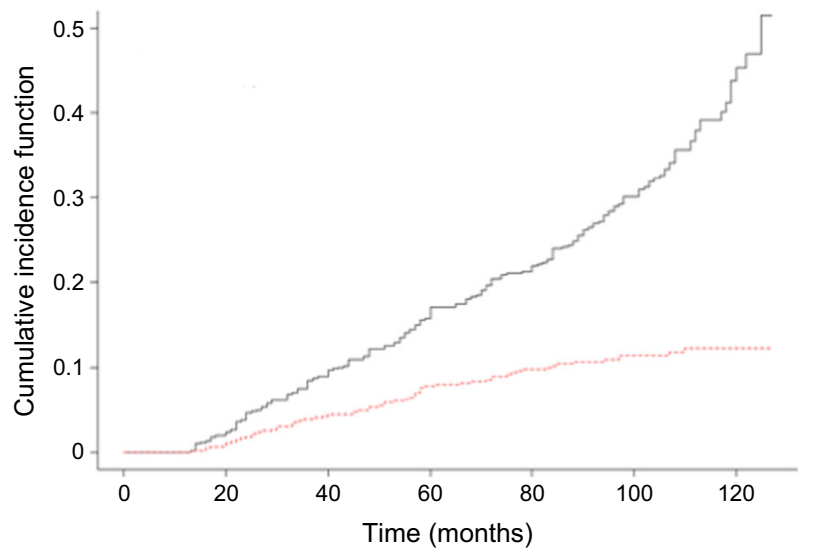

b

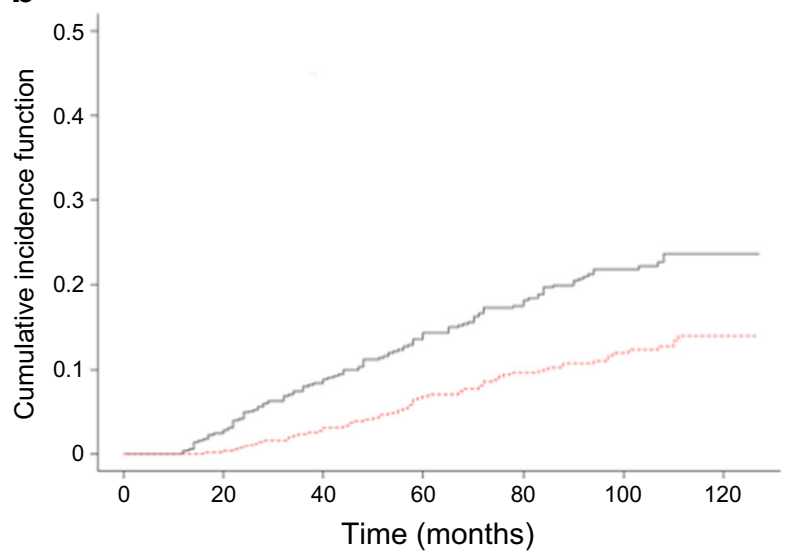

C

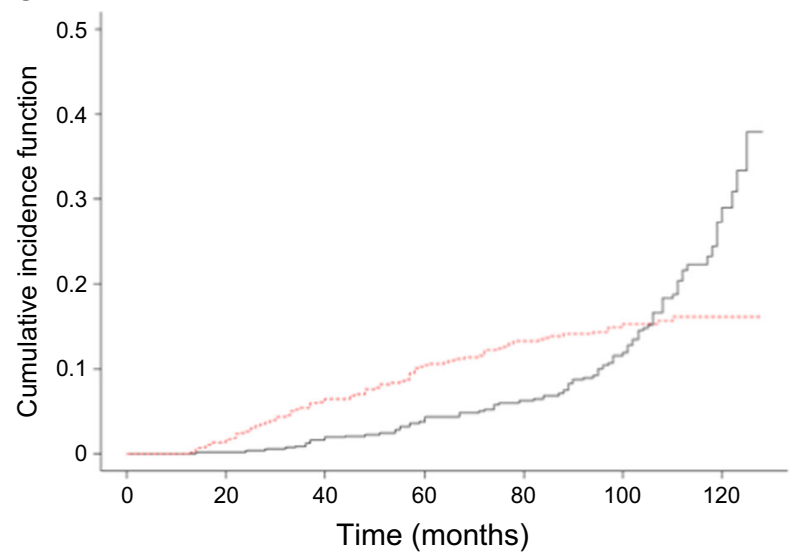

Fig. 1 Cumulative incidence function, calculated by the Fine and Gray competing risks analysis, of the composite renal outcome (a), microalbuminuria development or progression (b) and renal failure outcomes (c) (grey solid lines), with their respective competing event of nonrenal failure deaths (red dashed lines). Time 0 represents the patients' entry into the study and the beginning of follow-up

Independent predictors of renal outcomes Table 2 presents the results of the multivariate competing risks and Cox analyses for the independent predictors of any renal event 
Table 1

Characteristics of all diabetic participants grouped according to composite renal outcome occurrence during follow-up

Characteristic

\section{All participants \\ Participants without}

$$
(n=629)
$$

Age, years

Male sex, \%

BMI, kg/m²

Smoking, current/past, \%

Physical activity, $\%$ of active

Diabetes duration, years

Chronic diabetes complications, $\%$

Cerebrovascular disease

Coronary artery disease

Peripheral arterial disease

Retinopathy

DKD

\section{AER $\geq 30 \mathrm{mg} / 24 \mathrm{~h}$}

eGFR 60-30 ml min ${ }^{-1} 1.73 \mathrm{~m}^{-2}$

Peripheral neuropathy

Cardiovascular autonomic neuropathy

Diabetes treatment, \%

Metformin

Sulfonylureas

Insulin

Dyslipidaemia, \%

Statin use, \%

Arterial hypertension, \%

No. of antihypertensive drugs

ACEi/ARB, \%

Diuretic, \%

Calcium channel blocker, $\%$

Beta blocker, \%

Clinic BP (mmHg)

Baseline SBP

Baseline DBP

Mean first-year SBP

Mean first-year DBP

Mean second-year SBP

Mean second-year DBP

Mean cumulative SBP

Mean cumulative DBP

Ambulatory BP ( $\mathrm{mmHg}$ )

$24 \mathrm{~h} \mathrm{SBP}$

$24 \mathrm{~h} \mathrm{DBP}$

Daytime SBP

Daytime DBP

Night-time SBP

Night-time DBP

Nocturnal SBP fall, $\%$ of daytime SBP

Normal SBP dipping pattern, $\%$

Nocturnal DBP fall, $\%$ of daytime DBP

$$
60.0 \pm 9.6
$$

37.7

$29.6 \pm 4.9$

45.5

23.3

$8(3-15)$

9.4

16.3

17.6

30.9

31.5

26.4

17.5

28.4

24.7

86.8

43.7

48.5

87.5

76.9

90.1

3 (1-3)

81.7

62.9

26.9

46.3

$\begin{array}{ll}147 \pm 25 & 146 \pm 24 \\ 84 \pm 13 & 84 \pm 13 \\ 140 \pm 19 & 139 \pm 18 \\ 79 \pm 11 & 79 \pm 10 \\ 141 \pm 19 & 138 \pm 18 \\ 78 \pm 11 & 77 \pm 10 \\ 139 \pm 16 & 137 \pm 14 \\ 77 \pm 9 & 77 \pm 9 \\ & \\ 129 \pm 15 & 127 \pm 14 \\ 74 \pm 10 & 73 \pm 9 \\ 131 \pm 15 & 129 \pm 14 \\ 75 \pm 10 & 75 \pm 10 \\ 121 \pm 18 & 120 \pm 18 \\ 69 \pm 11 & 68 \pm 11 \\ 7.3 \pm 8.6 & 7.1 \pm 8.9 \\ 42.4 & 42.4 \\ 8.7 \pm 9.5 & 8.4 \pm 9.7\end{array}$

renal outcomes

( $n=434)$

$60.2 \pm 9.9$
36.9
$29.5 \pm 4.9)$
43.8
23.9
$7(3-15)$

8.9

17.0

16.6

27.3

27.1

23.4

13.4

25.7

21.2

89.4

45.2

45.9

86.9

76.6

88.9

$3(1-3)$

80.5

62.2

25.5

45.0

$8.4 \pm 9.7$
Participants with renal outcomes $(n=195)$

$\begin{array}{ll}59.9 \pm 9.1 & 0.72 \\ 39.5 & 0.54 \\ 29.7(4.7) & 0.71 \\ 49.2 & 0.21 \\ 22.1 & 0.62 \\ 9(4-16) & 0.047\end{array}$

10.3

14.9

20.0

38.9

40.5

32.8

26.7

38.5

32.0

81.0

40.5

54.4

88.7

77.4

92.8

3 (2-4)

84.3

64.6

30.3

49.2

$150 \pm 26$

$84 \pm 14$

$144 \pm 22$

$79 \pm 11$

$146 \pm 21$

$79 \pm 12$

$144 \pm 17$

$79 \pm 11$

$132 \pm 17$

$75 \pm 11$

$134 \pm 17$

$77 \pm 11$

$124 \pm 18$

$70 \pm 11$

$7.6 \pm 7.9$

42.5

$9.4 \pm 9.2$ $p$ value

(renal outcome vs no renal outcome)

0.72

0.54

0.71

0.21

0.047

0.60

0.51

0.29

0.004

0.001

0.014

$<0.001$

0.001

0.007

0.004

0.27

0.049

0.53

0.82

0.38

0.043

0.044

0.56

0.21

0.32

0.09

0.99

0.002

0.65

$<0.001$

0.07

$<0.001$

0.20

$<0.001$

0.024

$<0.001$

0.011

0.010

0.20

0.52

0.99

0.23 
Table 1 (continued)

\begin{tabular}{|c|c|c|c|c|}
\hline Characteristic & $\begin{array}{l}\text { All participants } \\
(n=629)\end{array}$ & $\begin{array}{l}\text { Participants without } \\
\text { renal outcomes } \\
(n=434)\end{array}$ & $\begin{array}{l}\text { Participants with } \\
\text { renal outcomes } \\
(n=195)\end{array}$ & $\begin{array}{l}p \text { value } \\
\text { (renal outcome vs } \\
\text { no renal } \\
\text { outcome) }\end{array}$ \\
\hline Normal DBP dipping pattern, $\%$ & 45.5 & 45.2 & 46.1 & 0.86 \\
\hline \multicolumn{5}{|l|}{ Laboratory variables } \\
\hline Fasting glucose, $\mathrm{mmol} / \mathrm{l}$ & $8.96 \pm 3.94$ & $8.03 \pm 4.04$ & $8.74 \pm 3.82$ & 0.35 \\
\hline Baseline $\mathrm{HbA}_{1 \mathrm{c}}, \%$ & $8.0 \pm 2.0$ & $8.0 \pm 1.9$ & $8.1 \pm 2.1$ & 0.35 \\
\hline Baseline $\mathrm{HbA}_{1 \mathrm{c}}, \mathrm{mmol} / \mathrm{mol}$ & $64 \pm 19.5$ & $64 \pm 18.4$ & $65 \pm 20$ & \\
\hline Mean first-year $\mathrm{HbA}_{1 \mathrm{c}}, \%$ & $7.7 \pm 1.5$ & $7.6 \pm 1.4$ & $7.9 \pm 1.7$ & 0.016 \\
\hline Mean first-year $\mathrm{HbA}_{1 \mathrm{c}}, \mathrm{mmol} / \mathrm{mol}$ & $61 \pm 14$ & $60 \pm 13$ & $63 \pm 16.2$ & \\
\hline Mean second-year $\mathrm{HbA}_{1 \mathrm{c}}, \%$ & $7.7 \pm 1.5$ & $7.6 \pm 1.4$ & $8.0 \pm 1.7$ & 0.009 \\
\hline Mean second-year $\mathrm{HbA}_{1 \mathrm{c}}, \mathrm{mmol} / \mathrm{mol}$ & $61 \pm 14$ & $60 \pm 13$ & $64 \pm 16.2$ & \\
\hline Mean cumulative $\mathrm{HbA}_{1 \mathrm{c}}, \%$ & $7.8 \pm 1.3$ & $7.7 \pm 1.3$ & $8.0 \pm 1.4$ & 0.002 \\
\hline Mean cumulative $\mathrm{HbA}_{1 \mathrm{c}}, \mathrm{mmol} / \mathrm{mol}$ & $62 \pm 11.9$ & $61 \pm 11.9$ & $64 \pm 13$ & \\
\hline Triacylglycerol, mmol/1 & $1.6(1.0-2.4)$ & $1.6(1.1-2.3)$ & $1.6(1.1-2.7)$ & 0.71 \\
\hline HDL-cholesterol, mmol/1 & $1.05 \pm 0.30$ & $1.10 \pm 0.30$ & $1.08 \pm 0.30$ & 0.43 \\
\hline LDL-cholesterol, mmol/l & $3.07 \pm 1.02$ & $3.07 \pm 1.02$ & $3.05 \pm 1.03$ & 0.82 \\
\hline eGFR, $\mathrm{ml} \mathrm{min} \min ^{-1} 1.73 \mathrm{~m}^{-2}$ & $92 \pm 33$ & $93 \pm 33$ & $88 \pm 34$ & 0.070 \\
\hline $\mathrm{AER}, \mathrm{mg} / 24 \mathrm{~h}$ & $14(7-43)$ & $11(7-33)$ & $21(9-101)$ & $<0.001$ \\
\hline Echocardiographic LVMI, g/m².7 & $52.5 \pm 16.9$ & $51.2 \pm 15.9$ & $55.2 \pm 18.7$ & 0.010 \\
\hline Echocardiographic LVH, \% & 60.8 & 59.9 & 62.7 & 0.51 \\
\hline Aortic stiffness (cf-PWV), m/s & $8.8 \pm 1.9$ & $8.6 \pm 1.8$ & $9.1 \pm 2.0$ & 0.004 \\
\hline Increased aortic stiffness (cf-PWV $>10 \mathrm{~m} / \mathrm{s}$ ), $\%$ & 23.0 & 21.1 & 27.0 & 0.11 \\
\hline
\end{tabular}

Data are expressed as proportions, means \pm SD or medians (interquartile range)

AER, albumin excretion rate; LHV, left ventricular hypertrophy (for women $>44 \mathrm{~g} / \mathrm{m}^{2.7}$; for men $>48 \mathrm{~g} / \mathrm{m}^{2.7}$ ); LVMI, left ventricular mass index

occurrence (the composite endpoint), new development or progression of albuminuria and renal failure outcomes. Mean first-year $\mathrm{HbA}_{1 \mathrm{c}}$, ambulatory $\mathrm{BP}$ and aortic stiffness were the main predictors of renal outcomes. Mean $\mathrm{HbA}_{1 \mathrm{c}}$ level was a significant predictor in Cox analyses but its predictive importance attenuated towards borderline significance in competing risks analyses. Otherwise, aortic stiffness was a significant predictor of microalbuminuria development/ progression and ambulatory SBP was a significant predictor of renal failure outcomes, both in Cox and in competing risks analyses. No evidence of interaction (all $p>0.20$ ) was observed between any independent predictor and baseline albumin excretion rate and eGFR, suggesting that the potential protective effect of better blood glucose and BP control on renal outcomes was not influenced by abnormal albuminuria or worsening renal function.

Table 3 shows the predictive strength of several BP variables for the three renal outcomes, adjusted for the other independent predictors and potential confounders in the competing risks framework. On the whole, systolic BP was a better predictor than diastolic BP. At baseline, ambulatory BP was a better predictor than BP measured in the clinic. Mean cumulative clinic SBP during follow-up presented the strongest association with the adverse renal outcomes, although baseline ambulatory SBP was a predictor equal to cumulative clinic SBP for worsening of renal function. The nocturnal fall in $\mathrm{BP}$ was not associated with any renal endpoint occurrence. We found no evidence of interaction between $\mathrm{HbA}_{1 \mathrm{c}}$ levels and any of the BP variables (all $p>0.20$ for interaction terms), suggesting that their effects on renal outcomes were independent and additive on the log scale. Electronic supplementary material (ESM) Table 1 shows similar results using classic Cox analysis.

In sensitivity analysis excluding individuals with preexistent DKD at baseline, increasing aortic stiffness still predicted development of microalbuminuria (HR 1.34; 95\% CI $1.01,1.78 ; p=0.044$ for increment of $1 \mathrm{SD}$ in cf.-PWV) and ambulatory SBPs still predicted renal function worsening (24 h SBP: HR 1.85; 95\% CI 0.97, 3.52; $p=0.063$; daytime SBP: HR 1.49; 95\% CI 1.04, 2.12; $p=0.029$ ). In the same way, after excluding individuals with endpoint occurrence during the first 3 years of follow-up, ambulatory BPs still predicted renal failure outcomes (24 h SBP: HR 1.59; $95 \%$ CI $1.00,2.50 ; p=0.048$; daytime SBP: HR 1.44; $95 \%$ CI 1.14 , $1.82 ; p=0.002$ ), as did mean first-year clinic SBP (HR 1.30; $95 \%$ CI $1.01,1.68 ; p=0.041$ ), suggesting that there was no 
Table 2 Independent predictors of renal outcomes in participants with type 2 diabetes after a median follow-up of 7.7 years

\begin{tabular}{|c|c|c|c|c|c|c|}
\hline \multirow[t]{2}{*}{ Independent predictor } & \multicolumn{2}{|c|}{$\begin{array}{l}\text { Composite renal outcome } \\
\text { (195 events) }\end{array}$} & \multicolumn{2}{|c|}{$\begin{array}{l}\text { Albuminuria development } \\
\text { or progression } \\
\text { (125 events) }\end{array}$} & \multicolumn{2}{|c|}{$\begin{array}{l}\text { Renal failure outcomes } \\
\text { (91 events) }\end{array}$} \\
\hline & $\operatorname{HR}(95 \% \mathrm{CI})$ & $p$ value & $\operatorname{HR}(95 \% \mathrm{CI})$ & $p$ value & $\operatorname{HR}(95 \% \mathrm{CI})$ & $p$ value \\
\hline \multicolumn{7}{|l|}{ Competing risks analysis } \\
\hline Mean first-year $\mathrm{HbA}_{1 \mathrm{c}}$ & $1.17(0.98,1.40)$ & 0.09 & $1.21(0.95,1.54)$ & 0.13 & $1.25(1.00,1.57)$ & 0.052 \\
\hline Ambulatory daytime SBP & $1.28(1.09,1.50)$ & 0.003 & $1.17(0.96,1.44)$ & 0.12 & $1.32(1.09,1.60)$ & 0.005 \\
\hline Aortic stiffness (cf-PWV) & $1.16(1.00,1.34)$ & 0.052 & $1.26(1.02,1.56)$ & 0.036 & $1.08(0.88,1.33)$ & 0.44 \\
\hline \multicolumn{7}{|l|}{ Cox survival analysis } \\
\hline Mean first-year $\mathrm{HbA}_{1 \mathrm{c}}$ & $1.22(1.04,1.44)$ & 0.018 & $1.26(1.03,1.55)$ & 0.022 & $1.27(1.02,1.59)$ & 0.036 \\
\hline Ambulatory daytime SBP & $1.29(1.12,1.49)$ & $<0.001$ & $1.18(0.98,1.43)$ & 0.080 & $1.41(1.16,1.71)$ & 0.001 \\
\hline Aortic stiffness (cf-PWV) & $1.15(0.99,1.34)$ & 0.066 & $1.26(1.04,1.53)$ & 0.018 & $1.06(0.85,1.32)$ & 0.59 \\
\hline
\end{tabular}

Data from competing risks (Fine and Gray approach) and Cox analyses are shown and are expressed as subdistribution HR (95\% CI) for competing risks analysis and as HR $(95 \% \mathrm{CI})$ for Cox analysis, estimated for increments of $1 \mathrm{SD}$ in each predictive variable

Candidate variables for entering the predictive models were as follows: BMI, smoking status, physical activity, dyslipidaemia, arterial hypertension, glucose-lowering treatment (metformin and insulin), antihypertensive treatment (number and classes of drugs), baseline clinic and ambulatory $24 \mathrm{~h}$ SBP, mean first-year $\mathrm{HbA}_{1 \mathrm{c}}$, each macrovascular (coronary, cerebrovascular and peripheral arterial disease) and microvascular (retinopathy and neuropathy) diabetic complication and aortic stiffness. All models were further adjusted for age, sex, diabetes duration, baseline albumin excretion rate and eGFR, and use of an ACEi or ARB

reverse causality between baseline and first-year BP and renal function worsening.

\section{Discussion}

This study has novel and clinically important findings. It demonstrated, as far as we know for the first time in type 2 diabetes using a competing risks analysis, that at baseline ambulatory $\mathrm{BP}$ was a better predictor than clinic BP for assessing the risk of DKD development and progression. This was particularly evident for clinical renal failure outcomes. Further, it demonstrated for the first time in an occidental population the prognostic importance of increased aortic stiffness to abnormal albuminuria development/progression, revealing potential new relationships between macro- and microvascular disease in type 2 diabetes. Overall, our findings not only emphasise the pivotal role of optimising control of blood glucose and BP in the prevention of DKD development/progression, but also provided strong support for more widespread use of ambulatory BP monitoring and aortic stiffness assessment in clinical diabetes management.

There have only been two previous studies [23, 24], in Asian populations, that investigated the associations between aortic stiffness and renal outcomes. The first [23], involving 461 Japanese individuals with type 2 diabetes followed-up for a median of 5.9 years, demonstrated the prognostic value of aortic stiffness for abnormal albuminuria development/ progression and reported an association with eGFR decline. However, this study enrolled only hospitalised patients with high baseline $\mathrm{HbA}_{1 \mathrm{c}}$ (around 9.0\% [74.9 mmol/mol]) and did not adjust for some important confounders, such as diabetes duration. The second of these studies [24], involving 577 Taiwanese individuals followed for only 1 year, reported associations between arterial stiffness and eGFR decline. However, the use of a method that measured mainly peripheral arterial stiffness (the brachial-ankle pulse wave velocity), the very short follow-up and the lack of statistical adjustment for several important confounders, such as diabetes duration, BP levels and albumin excretion rate, severely compromised its findings. Our study examined a larger sample of outpatients, followed them for a longer period and used a more comprehensive statistical adjustment in a competing risks setting. We confirmed the prognostic importance of increased aortic stiffness, measured by its gold-standard (the cf-PWV [30]), in predicting future development/progression of abnormal albuminuria in an occidental population with type 2 diabetes. The previous studies [23, 24] found associations between arterial stiffness and the annual rate of eGFR decline, whereas we did not find any association with clinical renal failure outcomes. In the two earlier analyses, however, the rate of eGFR decline and classic clinical renal failure outcomes are different endpoints and not comparable or interchangeable at all. On the other hand, it is well-established that a significant proportion of diabetic individuals develop advanced renal failure without albuminuria development or progression [2, 32]. Indeed, in our cohort, of the 91 individuals who experienced one of the renal failure outcomes, abnormal albuminuria only developed or progressed concomitantly in 21 (23\%). Hence, it was not unexpected to observe that increased aortic stiffness was a major predictor of 
Table 3 Predictive value of clinic and ambulatory BP variables measured at different time intervals during follow-up for the three renal outcomes, estimated by competing risks analysis (Fine and Gray approach)

\begin{tabular}{|c|c|c|c|c|c|c|}
\hline \multirow[t]{2}{*}{ Variable } & \multicolumn{2}{|c|}{$\begin{array}{l}\text { Composite renal outcome } \\
\text { (195 events) }\end{array}$} & \multicolumn{2}{|c|}{$\begin{array}{l}\text { Albuminuria development or progression } \\
\text { (125 events) }\end{array}$} & \multicolumn{2}{|c|}{$\begin{array}{l}\text { Renal failure outcomes } \\
\text { (91 events) }\end{array}$} \\
\hline & $\operatorname{HR}(95 \% \mathrm{CI})$ & $p$ value & HR $(95 \%$ CI) & $p$ value & $\operatorname{HR}(95 \% \mathrm{CI})$ & $p$ value \\
\hline \multicolumn{7}{|l|}{ SBP } \\
\hline Baseline clinic & $1.05(0.89,1.25)$ & 0.56 & $1.14(0.93,1.40)$ & 0.22 & $1.00(0.78,1.26)$ & 0.96 \\
\hline Mean first-year clinic & $1.23(1.04,1.44)$ & 0.014 & $1.18(0.97,1.44)$ & 0.10 & $1.19(0.95,1.50)$ & 0.13 \\
\hline Mean second-year clinic & $1.31(1.12,1.52)$ & $<0.001$ & $1.31(1.07,1.59)$ & 0.009 & $1.24(1.01,1.52)$ & 0.044 \\
\hline Mean cumulative clinic & $1.39(1.18,1.65)$ & $<0.001$ & $1.31(1.06,1.62)$ & 0.014 & $1.30(1.05,1.62)$ & 0.018 \\
\hline Ambulatory $24 \mathrm{~h}$ & $1.33(0.98,1.80)$ & 0.070 & $1.10(0.74,1.65)$ & 0.64 & $1.55(1.01,2.40)$ & 0.048 \\
\hline Ambulatory daytime & $1.28(1.09,1.50)$ & 0.003 & $1.17(0.96,1.44)$ & 0.12 & $1.32(1.09,1.60)$ & 0.005 \\
\hline Ambulatory night-time & $1.13(0.99,1.31)$ & 0.080 & $1.05(0.87,1.26)$ & 0.63 & $1.25(1.04,1.50)$ & 0.018 \\
\hline Nocturnal fall & $1.10(0.95,1.28)$ & 0.22 & $1.13(0.93,1.37)$ & 0.21 & $1.00(0.81,1.23)$ & 0.99 \\
\hline \multicolumn{7}{|l|}{ DBP } \\
\hline Baseline clinic & $0.94(0.81,1.10)$ & 0.44 & $0.98(0.80,1.19)$ & 0.80 & $0.94(0.74,1.19)$ & 0.58 \\
\hline Mean first-year clinic & $0.96(0.81,1.14)$ & 0.64 & $0.90(0.73,1.12)$ & 0.36 & $1.00(0.78,1.27)$ & 0.98 \\
\hline Mean second-year clinic & $1.12(0.95,1.33)$ & 0.18 & $1.02(0.82,1.26)$ & 0.88 & $1.27(1.01,1.58)$ & 0.038 \\
\hline Mean cumulative clinic & $1.23(1.03,1.47)$ & 0.024 & $1.15(0.92,1.45)$ & 0.22 & $1.18(0.93,1.50)$ & 0.17 \\
\hline Ambulatory $24 \mathrm{~h}$ & $1.18(1.00,1.38)$ & 0.050 & $1.09(0.88,1.34)$ & 0.44 & $1.28(1.04,1.58)$ & 0.022 \\
\hline Ambulatory daytime & $1.20(1.03,1.40)$ & 0.024 & $1.09(0.89,1.33)$ & 0.41 & $1.30(1.05,1.61)$ & 0.017 \\
\hline Ambulatory night-time & $1.07(0.92,1.25)$ & 0.39 & $0.98(0.80,1.20)$ & 0.82 & $1.20(0.99,1.47)$ & 0.068 \\
\hline Nocturnal fall & $1.11(0.96,1.29)$ & 0.16 & $1.15(0.95,1.39)$ & 0.15 & $1.02(0.81,1.29)$ & 0.85 \\
\hline
\end{tabular}

Data are expressed as subdistribution HR (95\% CI) (Fine and Gray approach) for competing risks analysis, estimated for increments of 1 SD in each predictive variable

All models were adjusted for age, sex, diabetes duration, presence of diabetic kidney disease at baseline (albumin excretion rate and eGFR), use of ACEi or ARB, aortic stiffness and $\mathrm{HbA}_{1 \mathrm{c}}$ level

microalbuminuria development, the classic physiopathological pathway of diabetic nephropathy, but not of ESRD outcomes, which probably involve other physiopathological mechanisms, such as metabolic, inflammatory and haemodynamic factors [2, $32,33]$. Interestingly, we have recently shown that increased aortic stiffness also predicted the development and worsening of peripheral neuropathy, another common diabetic microvascular complication [25]. This supports the use of aortic stiffness as an intermediary surrogate marker for both macro- and microvascular complications in type 2 diabetes [34] and sheds light on the possible inter-relationships between macro- and microcirculatory abnormalities. Increased aortic stiffness, by losing the normal aortic buffering function on pulsatile forces, may increase the transmission of high, potentially damaging, pulsatile pressure waves to the microcirculation. This may be particularly hazardous in high-flow/low-resistance organs, such as the kidney, leading to endothelial dysfunction and damage, favouring the development of microalbuminuria [34].

It has been consistently demonstrated that $24 \mathrm{~h}$ ambulatory $\mathrm{BP}$ is better at predicting cardiovascular risk than BP measured in an office setting [14, 15, 28]. However, information is still scarce regarding its value for estimating the risk of renal endpoints in type 2 diabetes. Data mainly come from crosssectional studies that reported ambulatory BP thresholds associated with abnormal albuminuria in type 2 diabetes [26, 35]. Since the seminal study by Lurbe and colleagues [36] in type 1 diabetes, only two prospective studies [16, 17] have addressed the importance of ambulatory BP variables to renal outcomes in individuals with type 2 diabetes. These studies evaluated only microalbuminuria development/progression and both reported that the best ambulatory BP predictor was $24 \mathrm{~h}$ pulse pressure, which is a well-known surrogate for increased arterial stiffness, in agreement with our findings. In the present study, baseline ambulatory SBP was better than clinic SBP and was as important as mean cumulative SBP during follow-up for prediction of renal failure outcomes. However, for development/progression of microalbuminuria, we showed that the mean SBP achieved during follow-up was a stronger predictor than baseline BP. This is in accordance with a recent post hoc analysis of the Atherosclerosis Risk in Communities (ARIC) database, which demonstrated that the best prediction model of cardiovascular risk was obtained by using the mean cumulative BP during follow-up [37]. Otherwise, the importance of the nocturnal fall in $\mathrm{BP}$ in 
addition to mean ambulatory BP for predicting DKD progression remains unclear in individuals with type 2 diabetes. We did not observe any prognostic value at all, either for microalbuminuria or for renal failure outcome. One of the previous studies reported similar findings [38], whereas the other [17] reported that fall in diastolic BP did contribute.

The importance of blood pressure control, particularly SBP, is largely established for prevention of DKD development and progression $[2,7,33]$. However, the optimal BP goal is still under debate. Although observational cohort studies, including the present one, support a continuous direct association between BP levels and renal outcomes risks; few interventional studies indeed achieved BP levels below 130/80 mmHg $[39,40]$. These studies suggested that reducing BP to these levels may prevent abnormal albuminuria development or progression, but did not lower clinical renal failure outcomes in relation to values around $135 / 85 \mathrm{mmHg}[7,8,10,12]$. Two recent meta-analyses $[7,10]$ confirmed that the best renal protection obtained by BP reduction was observed when individuals had clinic SBP >140 $\mathrm{mmHg}$ at baseline and achieved mean SBP during follow-up between $<140$ and $\geq 130 \mathrm{mmHg}$. Reducing SBP to $<130 \mathrm{mmHg}$ only prevented progression of abnormal albuminuria [7] and did not improve ESRD outcomes [7, 10].

The importance of optimal blood glucose control in preventing the development and progression of abnormal albuminuria is well accepted and indeed is one of the main factors recommended in the prevention and management of DKD $[2,32,33]$. However, the impact of strict blood glucose control on ESRD outcomes is still debatable. The Action in Diabetes and Vascular Disease: Preterax and Diamicron MR Controlled Evaluation (ADVANCE) trial in type 2 diabetes [6, 13] and the Diabetes Control and Complications Trial/ Epidemiology of Diabetes Interventions and Complications (DCCT/EDIC) in type 1 diabetes [41] both found a protective effect; the Action to Control Cardiovascular Risk in Diabetes (ACCORD) [12] and the Veterans Affairs Diabetes Trial (VADT) [42] and two meta-analyses in type 2 diabetes [5, 9] all showed no effect on ESRD outcomes, although all these studies recognised their low statistical power because of the low incidence of this endpoint due possibly to short follow-up periods. We showed that a higher mean $\mathrm{HbA}_{1 \mathrm{c}}$ during the first year of follow-up, which was when greatest $\mathrm{HbA}_{1 \mathrm{c}}$ reduction occurred, was predictor of both outcomes in classic Cox proportional hazards analysis, although the competing risks analysis attenuated its predictive capacity to non-significant values for adverse renal outcomes. However, it should be noted that the Cox and competing risks analyses provide two different risk estimands.

There are some limitations of this study that should be addressed. First, this study enrolled middle-aged to elderly high-risk individuals with long-standing type 2 diabetes at a tertiary-care institution. Therefore, our findings may not be generalised to younger individuals with recent-onset diabetes or individuals who are undergoing primary care management. Second, it is an observational cohort so neither cause-andeffect relationships nor physiopathological mechanisms can be inferred, but only speculated. Moreover, although we negated the possibility of reverse causality between increased baseline and first-year BP values and DKD development/progression, it still might have influenced particularly the associations between cumulative SBP during follow-up and renal outcomes, where increasing BP might be a consequence of DKD progression, instead of its predictor. Third, we did not address any correction for multiple comparisons. However, if we had applied Bonferroni's correction, since we had three outcomes for each predictor (the composite endpoint and its two separate components), we would have considered a $p$ value $<0.02$ as significant. With this more conservative threshold, only baseline ambulatory BP and follow-up clinic BP would not be considered significant predictors. On the other hand, the main strength is that our well-documented cohort allowed us to perform a comprehensive competing risks analysis of possible factors that could have an impact on the associations with renal outcomes. Furthermore, all participants underwent serial $24 \mathrm{~h}$ albumin excretion rate measurement at baseline and during follow-up, which is considered the gold-standard method.

In conclusion, this prospective observational cohort study demonstrated that baseline ambulatory systolic BP and aortic stiffness were important predictors of diverse renal outcomes in high-risk individuals with type 2 diabetes and should be included into their routine clinical management. Whether specifically targeting ambulatory BP and attenuating aortic stiffness, beyond the recommended clinic BP and blood glucose control, will be able to prevent or delay development or progression of DKD shall be the focus of future intervention studies.

Data availability The Rio de Janeiro Type 2 Diabetes Cohort Study is an ongoing study, and its dataset is not publicly available to ensure that the privacy of individual participants is maintained. However, data may be available from the corresponding author on reasonable request.

Funding This study was supported by grants from the Conselho Nacional de Desenvolvimento Científico e Tecnológico (CNPq, Brazil) and from the Fundação Carlos Chagas Filho de Amparo a Pesquisa do Estado do Rio de Janeiro (FAPERJ, Brazil). The sponsors had no role in study design, collection and analysis of data, interpretation of results or preparation, review and approval of the manuscript.

Duality of interest The authors declare that there is no duality of interest associated with this manuscript.

Contribution statement CRLC, NCL and GFS conceived and designed the study, followed-up the participants and obtained the data. MTF performed all aortic stiffness measurements. GCS performed the competing risks analyses. CRLC drafted the manuscript. GFS analysed 
the data and is the guarantor. All authors helped interpret the results, contributed with intellectual content, reviewed the manuscript and approved the final version to be published. GFS had full access to all the data and takes responsibility for the integrity of the data and the accuracy of data analysis.

\section{References}

1. Zimmet PZ, Magliano DJ, Herman WH, Shaw JE (2014) Diabetes: a 21st century challenge. Lancet Diabetes Endocrinol 2:56-64

2. Macisaac RJ, Ekinci EI, Jerums G (2014) Markers of and risk factors for the development and progression of diabetic kidney disease. Am J Kidney Dis 63(2 Suppl 2):S39-S62

3. Jha V, Garcia-Garcia G, Iseki K et al (2013) Chronic kidney disease: global dimension and perspectives. Lancet 382:260-272

4. Afkarian M, Sachs MC, Kestenbaum B et al (2013) Kidney disease and increased mortality risk in type 2 diabetes. J Am Soc Nephrol 24:302-308

5. Coca SG, Ismail-Beigi F, Haq N, Krumholz HM, Parikh CR (2012) Role of intensive glucose control in development of renal end points in type 2 diabetes mellitus: systematic review and metaanalysis intensive glucose control in type 2 diabetes. Arch Intern Med 172:761-769

6. Perkovic V, Heerspink HL, Chalmers J et al (2013) Intensive glucose control improves kidney outcomes in patients with type 2 diabetes. Kidney Int 83:517-523

7. Emdin CA, Rahimi K, Neal B, Callender T, Perkovic V, Patel A (2015) Blood pressure lowering in type 2 diabetes: a systematic review and meta-analysis. JAMA 313:603-615

8. Anderson RJ, Bahn GD, Emanuele NV, Marks JB, Duckworth WC, VADT Study Group (2014) Blood pressure and pulse pressure effects on renal outcomes in the veterans affairs diabetes trial (VADT). Diabetes Care 37:2782-2788

9. Boussageon R, Bejan-Angoulvant T, Saadatian-Elahi M et al (2011) Effect of intensive glucose lowering treatment on all cause mortality, cardiovascular death, and microvascular events in type 2 diabetes: meta-analysis of randomised controlled trials. BMJ 343: d4169

10. Brunström M, Carlberg B (2016) Effect of antihypertensive treatment at different blood pressure levels in patients with diabetes mellitus: systematic review and meta-analyses. BMJ 352:i717

11. Zoungas S, de Galan BE, Ninomiya Tet al (2009) Combined effects of routine blood pressure lowering and intensive glucose control on macrovascular and microvascular outcomes in patients with type 2 diabetes: new results from the ADVANCE. Diabetes Care 32:2068 2074

12. Ismail-Beigi F, Craven TE, O'Connor PJ et al (2012) Combined intensive blood pressure and glycemic control does not produce an additive benefit on microvascular outcomes in type 2 diabetic patients. Kidney Int 81:586-594

13. Wong MG, Perkovic V, Chalmers J et al (2016) Long-term benefits of intensive glucose control for preventing end-stage kidney disease: ADVANCE-ON. Diabetes Care 39:694-700

14. ABC-H Investigators, Roush GC, Fagard RH, Salles GF et al (2014) Prognostic impact from clinic, daytime, and night-time systolic blood pressure in nine cohorts of 13,844 patients with hypertension. J Hypertens 32:2332-2340

15. Salles GF, Leite NC, Pereira BB, Nascimento EM, Cardoso CR (2013) Prognostic impact of clinic and ambulatory blood pressure components in high-risk type 2 diabetic patients: the Rio de Janeiro Type 2 Diabetes Cohort Study. J Hypertens 31:2176-2186
16. Palmas W, Moran A, Pickering T et al (2006) Ambulatory pulse pressure and progression of urinary albumin excretion in older patients with type 2 diabetes mellitus. Hypertension 48:301-308

17. Knudsen ST, Laugesen E, Hansen KW, Bek T, Mogensen CE, Poulsen PL (2009) Ambulatory pulse pressure, decreased nocturnal blood pressure reduction and progression of nephropathy in type 2 diabetic patients. Diabetologia 52:698-704

18. Cavalcante JL, Lima JA, Redheuil A, Al-Mallah MH (2011) Aortic stiffness: current understanding and future directions. J Am Coll Cardiol 57:1511-1522

19. Kimoto E, Shoji T, Shinohara K et al (2003) Preferential stiffening of central over peripheral arteries in type 2 diabetes. Diabetes 52: $448-452$

20. Liu JJ, Tavintharan S, Yeoh LY et al (2014) High normal albuminuria is independently associated with aortic stiffness in patients with type 2 diabetes. Diabet Med 31:1199-1204

21. Cardoso CR, Ferreira MT, Leite NC, Barros PN, Conte PH, Salles GF (2009) Microvascular degenerative complications are associated with increased aortic stiffness in type 2 diabetic patients. Atherosclerosis 205:472-476

22. Sjöblom P, Nystrom FH, Länne T, Engvall J, Östgren CJ (2014) Microalbuminuria, but not reduced eGFR, is associated with cardiovascular subclinical organ damage in type 2 diabetes. Diabetes Metab 40:49-55

23. Bouchi R, Babazono T, Mugishima M et al (2011) Arterial stiffness is associated with incident albuminuria and decreased glomerular filtration rate in type 2 diabetic patients. Diabetes Care 34:2570 2575

24. Sheen YJ, Lin JL, Li TC, Bau CT, Sheu WH (2013) Peripheral arterial stiffness is independently associated with a rapid decline in estimated glomerular filtration rate in patients with type 2 diabetes. Biomed Res Int 2013:309294

25. Cardoso CR, Moran CB, Marinho FS, Ferreira MT, Salles GF (2015) Increased aortic stiffness predicts future development and progression of peripheral neuropathy in patients with type 2 diabetes: the Rio de Janeiro Type 2 Diabetes Cohort Study. Diabetologia 58:2161-2168

26. Cardoso CR, Leite NC, Muxfeldt ES, Salles GF (2012) Thresholds of ambulatory blood pressure associated with chronic complications in type 2 diabetes. Am J Hypertens 25:82-88

27. Levey AS, Stevens LA, Schmid CH et al (2009) A new equation to estimate glomerular filtration rate. Ann Intern Med 150:604-612

28. O'Brien E, Parati G, Stergiou G et al (2013) European Society of Hypertension position paper on ambulatory blood pressure monitoring. J Hypertens 31:1731-1768

29. Levey AS, Coresh J, Greene T et al (2007) Expressing the modification of diet in renal disease study equation for estimating glomerular filtration rate with standardized serum creatinine values. Clin Chem 53:766-772

30. Van Bortel LM, Laurent S, Boutouyrie P et al (2012) Expert consensus document on the measurement of aortic stiffness in daily practice using carotid-femoral pulse wave velocity. J Hypertens 30:445-448

31. Fine JP, Gray RJ (1999) A proportional hazards model for the subdistribution of a competing risk. J Am Stat Assoc 94:496-509

32. Macisaac RJ, Jerums G (2011) Diabetic kidney disease with and without albuminuria. Curr Opin Nephrol Hypertens 20:246-257

33. Radcliffe NJ, Seah JM, Clarke M, MacIsaac RJ, Jerums G, Ekinci EI (2017) Clinical predictive factors in diabetic kidney disease progression. J Diabetes Investig 8:6-18

34. Cardoso CR, Salles GF (2016) Aortic stiffness as a surrogate endpoint to micro- and macrovascular complications in patients with type 2 diabetes. Int J Mol Sci 17:2044

35. Moran A, Palmas W, Pickering TG et al (2006) Office and ambulatory blood pressure are independently associated with 
albuminuria in older subjects with type 2 diabetes. Hypertension 47:955-961

36. Lurbe E, Redon J, Kesani A et al (2002) Increase in nocturnal blood pressure and progression to microalbuminuria in type 1 diabetes. $\mathrm{N}$ Engl J Med 347:797-805

37. Sweeting MJ, Barrett JK, Thompson SG, Wood AM (2016) The use of repeated blood pressure measures for cardiovascular risk prediction: a comparison of statistical models in the ARIC study. Stat Med. https://doi.org/10.1002/sim.7144

38. Palmas W, Pickering T, Teresi J et al (2008) Nocturnal blood pressure elevation predicts progression of albuminuria in elderly people with type 2 diabetes. J Clin Hypertens (Greenwich) 10:12-20
39. The ACCORD Study Group (2010) Effects of intensive bloodpressure control in type 2 diabetes mellitus. N Engl J Med 362: $1575-1585$

40. Cooper-DeHoff RM, Gong Y, Handberg EM et al (2010) Tight blood pressure control and cardiovascular outcomes among hypertensive patients with diabetes and coronary artery disease. JAMA 304:61-68

41. The DCCT/EDIC Research Group (2011) Intensive diabetes therapy and glomerular filtration rate in type 1 diabetes. N Engl J Med 365:2366-2376

42. Agrawal L, Azad N, Emanuele NV et al (2011) Observation on renal outcomes in the Veterans Affairs Diabetes Trial. Diabetes Care 34:2090-2094 